\title{
Treinamento muscular ventilatório em pacientes tetraplégicos pós lesão medular traumática: revisão integrativa
}

\author{
Ventilatory muscle training in tetraplegic \\ patients after traumatic medal injury: \\ integration review
}

\author{
Bruno Souza de Matos ${ }^{1}$ \\ Andressa Santana Montino de Melo \\ Tatiana Maita Alves Conceição 3
}

\begin{abstract}
1Autor para correspondência. Centro Universitário Jorge Amado (Salvador). Bahia, Brasil. bruno_souza1801@hotmail.com 2,3Centro Universitário Jorge Amado (Salvador). Bahia, Brasil. andressamontinomelo@gmail.com, tatianamaita@bol.com.br
\end{abstract}

\begin{abstract}
RESUMO | INTRODUÇÃO: O Trauma Raquimedular é uma condição clínica incapacitante que pode desencadear a tetraplegia, descrita como paralisia parcial ou completa do tronco, músculos respiratórios e membros, favorecendo o surgimento de complicações, principalmente de caráter respiratório. Diante disso, o Fisioterapeuta pode utilizar treinamento muscular ventilatório(TMV) objetivando aumentar a força e resistência à fadiga dos músculos ventilatórios, além de prevenir complicações respiratórias. OBJETIVO: Descrever quais os dispositivos, protocolos e técnicas mais utilizadas para treinamento muscular ventilatório e os efeitos associados em indivíduos tetraplégicos pós trauma raquimedular. METODOLOGIA: Foi realizado levantamento bibliográfico entre novembro de 2019 e fevereiro de 2020, nas bases de dados Medline, LILACS e SciELO utilizando as palavraschave: Breathing Exercises, Spinal Cord Injuries, Quadriplegia e os sinônimos utilizando os operadores booleanos "AND" e "OR". RESULTADOS: Após consulta nas bases de dados, foram encontrados 3334 artigos, 36 foram selecionados para leitura de resumo, sucedendo que 23 foram excluídos por não atender aos critérios de seleção, restando 12 artigos para a leitura integral, resultando na seleção final de 12 artigos. CONCLUSÃo: Fica evidenciado que protocolos para TMV através de resistores lineares são os mais utilizados tetraplégicos. Além disso, execução de protocolos com resistores lineares a inspiração, empregando intensidade em torno de $30-60 \%$ da PImax, apresentou efeitos significativos em PImax. No entanto, percebeu-se que há muitas discordâncias quanto as variáveis de treinamento, principalmente intensidade e número de séries propostos. Contudo, é notório que a utilização do TMV em tetraplégicos apresenta certas divergências, principalmente relacionado a escolha das técnicas e/ou dispositivos adequados.
\end{abstract}

PALAVRAS-CHAVE: Exercícios respiratórios. Traumatismos da medula espinal. Quadriplegia.

\begin{abstract}
INTRODUCTION: Spinal trauma is a disabling clinical condition that can trigger quadriplegia, described as partial or complete paralysis of the trunk, respiratory muscles and limbs, favoring the onset of complications, mainly of a respiratory nature. Therefore, the Physiotherapist can use ventilatory muscle training (TMV) in order to increase the strength and fatigue resistance of the ventilatory muscles, in addition to preventing respiratory complications. OBJECTIVE: To describe which devices, protocols and techniques are most used for ventilatory muscle training and the associated effects in quadriplegic individuals after spinal cord trauma. METHODOLOGY: A bibliographic survey was carried out between November 2019 and February 2020, in the Medline, LILACS and SciELO databases using the keywords: Breathing Exercises, Spinal Cord Injuries, Quadriplegia and synonyms using the Boolean operators "AND" and "OR". RESULTS: After consulting the databases, 3334 articles were found, 36 were selected for reading the summary, with 23 being excluded for not meeting the selection criteria, leaving 12 articles for the full reading, resulting in the final selection of 12 articles. CONCLUSION: It is evidenced that protocols for TMV through linear resistors are the most used quadriplegics. In addition, execution of protocols with linear resistors to inspiration, using intensity around $30-60 \%$ of MIP, had significant effects on MIP. However, it was noticed that there are many disagreements regarding the training variables, mainly intensity and number of series proposed. However, it is clear that the use of TMV in quadriplegics presents certain divergences, mainly related to the choice of appropriate techniques and / or devices.
\end{abstract}

KEYWORDS: Breathing exercises. Spinal cord injuries. Quadriplegia. 


\section{Introdução}

O Trauma Raquimedular (TRM) é considerado pela Organização Mundial de Saúde (OMS) como um grave problema de saúde pública que ocorre em virtude de um evento traumático ocasionando lesão da medula espinhal ${ }^{1,2}$. A incidência mundial de TRM é de 10,5 casos por 100.000 pessoas, resultando em um número estimado de 768.473 novos casos de lesão medular anualmente em todo o mundo ${ }^{3,4}$. As manifestações clínicas dependem da extensão e localização da lesão. Essa condição clínica é incapacitante e gera disfunções autonômicas, perda parcial ou total da motricidade voluntária e/ou da sensibilidade (tátil, dolorosa, profunda), além de proporcionar disfunção dos músculos respiratórios ${ }^{3}$.

Imediatamente após uma lesão medular traumática, há um período de choque espinhal, resultando em paralisia flácida dos músculos abaixo do nível da lesão. Essa paralisia gera desequilíbrio e desvantagem mecânica resultando em ventilação menos eficiente, aumento do trabalho respiratório e uma tendência ao colapso das vias aéreas distais e à microatelectasia, provocando disfunções respiratórias. Estas repercussões respiratórias apresentam-se como causa mais comum de mortalidade após a lesão medular ${ }^{5,6}$ e são responsáveis por cerca de $80 \%$ dos óbitos em pacientes hospitalizados com lesão cervical, sendo que a pneumonia é responsável por $50 \%$ deles 7 .

No âmbito multiprofissional de assistência respiratória, o fisioterapeuta pode empregar um conjunto de procedimentos e técnicas que possibilitem melhor reabilitação respiratória. Dentre eles, destaca-se o treinamento muscular ventilatório (TMV), que objetiva melhorar ou redistribuir a ventilação, aumentar a força, resistência à fadiga e a coordenação dos músculos respiratórios, melhorar a capacidade da tosse e melhorar a capacidade funcional do paciente para atividades de vida diária. A indicação de fortalecimen- to muscular respiratório é avaliada através da identificação de fraqueza desses músculos ${ }^{8,9}$.

O objetivo dessa revisão integrativa foi descrever quais os dispositivos, protocolos e técnicas mais utilizadas para treinamento muscular ventilatório e os efeitos associados em indivíduos tetraplégicos pós trauma raquimedular.

\section{Metodologia}

Este estudo trata-se de uma Revisão Integrativa que é um método de revisão elaborado com fundamentação por meio de um processo mais amplo, pois abrange literatura teórica e empírica de modo que inclui estudos com diversos tipos de abordagens metodológicas que podem ser quantitativas e/ou qualitativas ${ }^{10,11}$. Para concepção desse artigo, foram consultadas as bases de dados eletrônicas Scientific Electronic Library (SciELO), Literatura LatinoAmericana em Ciências de Saúde (LILACS), e PubMed/ MEDLINE. A busca foi realizada entre novembro de 2019 e Fevereiro de 2020.

Foram utilizadas as palavras-chave Breathing Exercises, Spinal Cord Injuries, Quadriplegia e os sinônimos de acordo com as bases de dados, identificados nos Descritores em Ciências da Saúde (DeCS) e no Medical Subject Headings (MeSH), utilizando os operadores booleanos "AND" e "OR" como mostra o Quadro 1. A seleção dos artigos deveria adequar-se aos seguintes critérios de inclusão: ano de publicação entre 2008 e 2020; abordar sobre o treinamento muscular ventilatório apenas em pacientes tetraplégicos pós-lesão medular, independente do nível acometido. Em relação aos critérios de exclusão: artigos que fugiram do tema ou que abordaram o treinamento muscular ventilatório em outros grupos de pacientes.

Quadro 1. Palavras-chave utilizadas na busca eletrônica acrescidas dos operadores booleanos "AND" e "OR" e respectiva combinação

\begin{tabular}{|l|l|}
\hline Palavras-chave & Sinônimos e /ou MeSH \\
\hline Breathing Exercises & Respiratory Muscle Training \\
\hline Spinal Cord Injuries & Spinal Cord Trauma \\
\hline Quadriplegia & Tetraplegia \\
\hline $\begin{array}{l}\text { ((((quadriplegia) OR (Tetraplegia)) AND (Spinal Cord Injuries)) AND (Breathing Exercises)) OR (Respiratory } \\
\text { Muscle Training) }\end{array}$ \\
\hline
\end{tabular}


No processo de seleção das evidências, a análise dos títulos e dos resumos encontrados na busca inicial de acordo aos critérios de elegibilidade estabelecidos. Caso o título e o resumo não fossem esclarecedores, o artigo seria consultado na íntegra. Após analisar criticamente e avaliar todos os estudos incluídos na revisão, os artigos foram apresentados em um quadro destacando os itens: autores, ano de publicação, desenho de estudo, número de participantes, grupos de comparação, descrição sucinta do protocolo de intervenção (duração, intensidade, frequência e intervalo entre sessões) e resumos dos resultados encontrados.

\section{Resultados}

A pesquisa nas bases de dados resultou em 3.334 artigos (Medline: 3.304; Lilacs:25; Scielo: 5), resultando em 3.311 registros após remover os duplicados. Destes, 3.275 artigos foram excluídos pelo título por não contemplarem os critérios de inclusão; 36 foram selecionados para leitura de resumo, sucedendo que 23 foram excluídos por não atender aos critérios de seleção, restando 12 artigos para a leitura integral. Destes, verificou-se que os 12 cumpriam todos os critérios de inclusão, resultando na seleção final de 12 artigos, conforme Figura 1. Os estudos incluídos contemplavam diferentes contextos clínicos e abrangiam diversos tipos de abordagens metodológicas, desde relato/série de caso a ensaios clínicos randomizados, conforme apresentado no Quadro 2.

Figura 1. Diagrama de fluxo da pesquisa

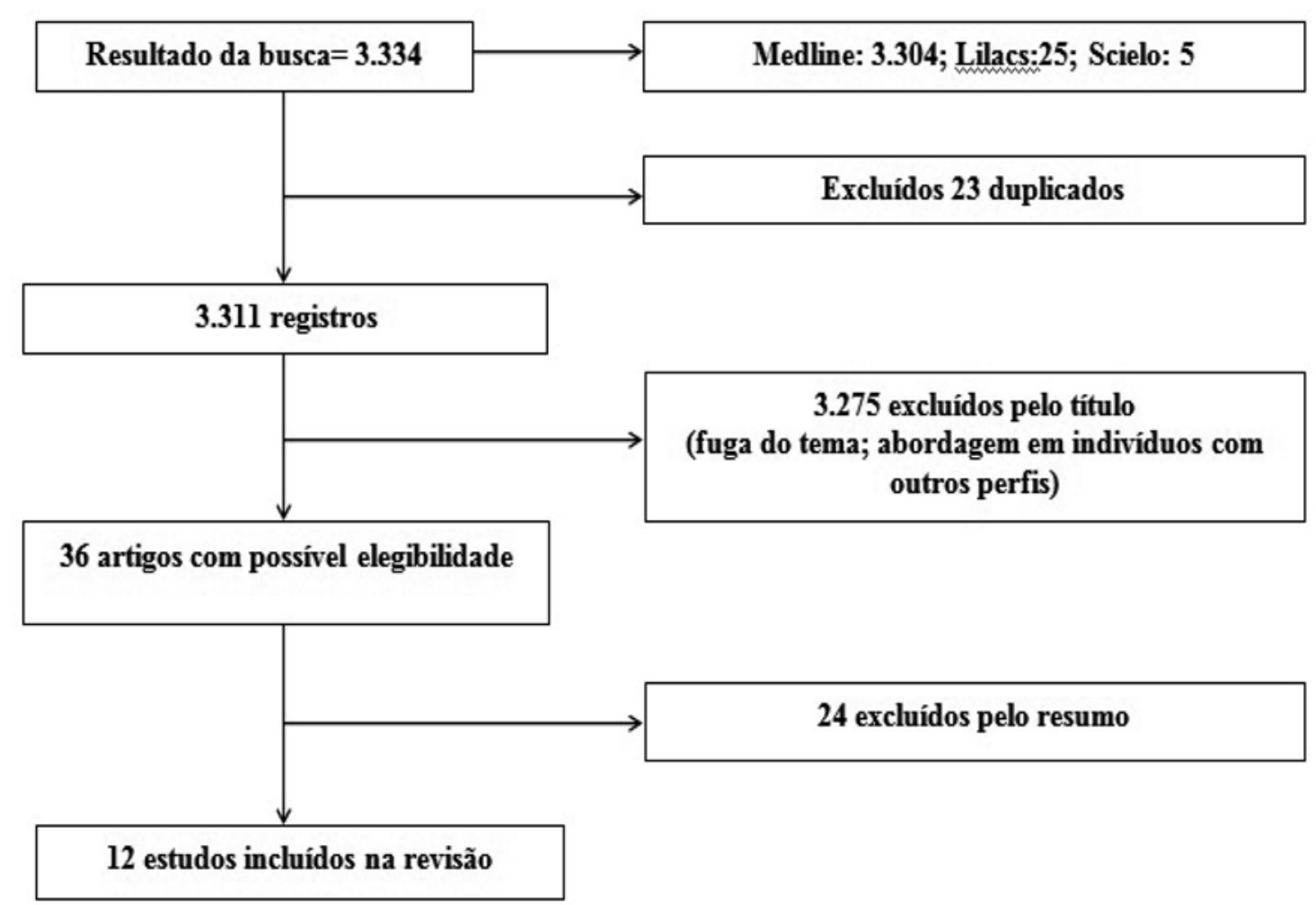

As publicações selecionadas apresentaram em seu conteúdo, a descrição dos dispositivos empregados. Dentre as possibilidades de intervenção encontradas, verificaram-se protocolos de treinamento com resistores de carga linear e alinear, hiperpneia normocápnica, assim como técnicas e dispositivos associados. Percebeu-se que um total de oito artigos $(66,6 \%)$ realizou os protocolos de intervenção, por meio de resistores de carga linear.

Um número de três estudos (25\%) realizou os protocolos de intervenção, por meio da associação entre Threshold $I M T \circledast$ (resistência à inspiração) e Threshold PEP $($ (resistência à expiração), descritos no Quadro 2. Nos estudos de Legg Ditterline et al. ${ }^{12}$ e Aslan et al. ${ }^{13}$, os dispositivos foram montados usando um sistema de válvulas de três vias com bocal, seguindo seus respectivos protocolos, enquanto o artigo de Boswell-Ruys et al. ${ }^{14}$ não forneceu relatos descritivos dos dispositivos utilizados, detalhando apenas o protocolo aplicado. 
Tendo em vista essas informações, verificou-se que a aplicação de protocolos associando o Threshold IMT® e Threshold $P E P \circledast$, proporcionou aumentos relacionados a força muscular inspiratória (PImáx) e expiratória (PEmáx), assim como a capacidade vital (CV), capacidade vital forçada (CVF), volume expiratório forçado no primeiro segundo (VEF1), o pico de fluxo expiratório (PEF) e, além de ganhos referentes a melhora do sono, redução da hipotensão ortostática e do índice apneia-hipopneia.

Ainda no que diz respeito à utilização de dispositivos com resistores de carga linear, observou-se que três artigos $15,16,17$ executaram seus protocolos por meio apenas do Threshold IMT® (25\%) em suas pesquisas (Quadro 2). Através dessas publicações, notou-se aumento da força muscular inspiratória em ambos os artigos e aumento da força muscular expiratória somente no estudo de Silveira et al. ${ }^{15}$, sem efeitos positivos em endurance.

No que diz respeito ao uso do PowerBreathe ${ }^{\circledR}$ para TMV, apenas um estudo executou protocolo utilizando esse dispositivo, conforme descrito no Quadro 2. Os autores apontam que esse dispositivo eletrônico de mão, relativamente recente, fornece uma carga resistiva de fluxo variável por meio de uma válvula controlada eletronicamente. Através desse protocoIo, McDonald e Stiller ${ }^{18}$ destacam que um programa de alta resistência e baixa repetição de TMV é viável e segura, sem que cause intercorrências registrados antes, durante ou após execução em adultos com lesão medular cervical completa ou torácica com função respiratória estável, na fase aguda.

O estudo de Russian et al. ${ }^{19}$ realizou o treinamento de resistência respiratória concomitante por meio de um equipamento que propõe resistência inspiratória e expiratória simultâneas, descrito comercialmente como PowerLung Performer ${ }^{\circledR}$. Este, assim como o de Boswell et al. ${ }^{15}$ foi um dos poucos artigos que avaliou os efeitos do TMV na função pulmonar, mas especificamente nos distúrbios do sono. Os resultados pós-treinamento indicaram melhorias na qualidade do sono, bem como redução da sonolência diurna, assim como aumento da PImax e PEmax (Quadro 2).

Ainda nesse contexto, Roth et al. ${ }^{20}$ conduziram sua pesquisa aplicando o treinamento por meio de resistor de carga alinear, especificamente o Medidor de Boehringer. Os autores avaliaram os efeitos treinamento muscular expiratório por intermédio do Medidor de Boehringer, um dispositivo com um pequeno tubo de plástico portátil onde os indivíduos expiram contra um manômetro. A resistência é dada pelo fato de ser um sistema fechado, criando resistência à alta pressão durante a expiração. Os resultados apresentados mostram que houve aumento estatisticamente significativo na variável PEmax $(p<0,02)$, apenas no grupo de treinamento de resistência, apresentados no quadro 2 .

Apenas Van Houtte et al. ${ }^{21}$ utilizaram a técnica de Hiperpneia Normocápnica para TMV. Esta técnica consiste em uma bolsa de respiração conectada a um sistema de tubo e bocal, com um pequeno orifício no tubo onde permite um fluxo inspiratório e expiratório adicional. O volume da bolsa é de cerca de 30 a 40\% da capacidade vital forçada do sujeito (CVF). Observou-se que a Ventilação Voluntária Máxima (VVM), a força e a resistência muscular respiratória melhoraram significativamente e as complicações respiratórias foram relatadas com menos frequência no grupo experimental em comparação com o grupo controle, conforme Quadro 2.

Dentre os estudos incluídos, dois realizaram o treinamento proposto, associando técnicas ou dispositivos diferentes (Quadro 2). Mueller et al. ${ }^{22}$ compararam os efeitos de um protocolo utilizando três técnicas diferentes: Treinamento muscular inspiratório por meio de resistor linear com feedback visual da resistência alcançada, comercialmente chamado de Respifit $\mathrm{S}{ }^{\circledR}$; Hiperpneia normocápnica através um dispositivo por nome comercial Spirotiger ${ }^{\circledR}$, que permite a hiperventilação intensiva através de uma nova respiração parcial do ar ventilado, apoiada por feedback visual; Além do grupo que realizou espirometria de incentivo com um dispositivo conhecido como Voldyne $5000 \AA$.

Em sua pesquisa, Kim et al. ${ }^{23}$ executaram seus protocolos por meio da associação da espirometria de incentivo e a manobra de tração abdominal. Em relação a espirometria de incentivo, eles afirmam ser uma das técnicas atuais utilizadas para treinar músculos respiratórios. Quanto a manobra de tração abdominal, descrevem que esta técnica é projetada para ativar o músculo transverso do abdômen (músculo envolvido na expiração forçada), sendo comumente usada em programas de treinamento de estabilização lombar. Para execução da manobra, foi utilizada uma unidade de biofeedback de pressão, com nome comercial Stabilizer ${ }^{\circledR}$, inflado para $50 \mathrm{mmHg}$, colocado entre a mesa de tratamento e o abdômen inferior dos indivíduos na posição prona, posteriormente solicitados a manter uma pressão inferior a $6-10 \mathrm{mmHg}$, com base no feedback visual de um manômetro analógico. 


\begin{tabular}{|c|c|c|c|c|}
\hline 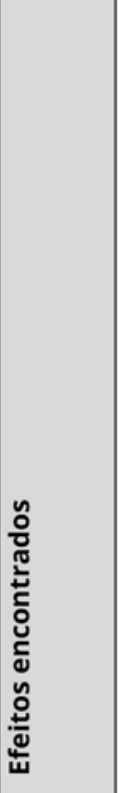 & 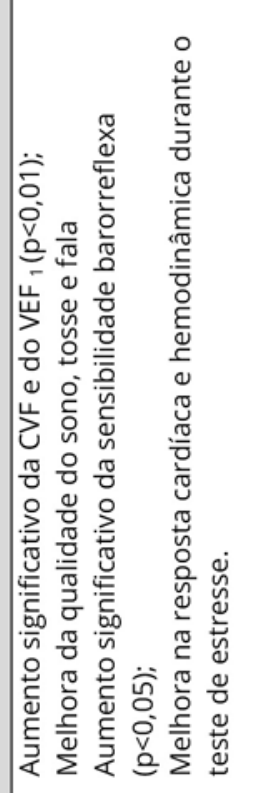 & 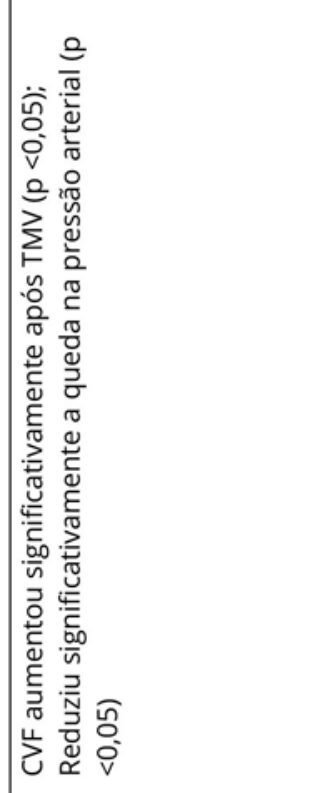 & 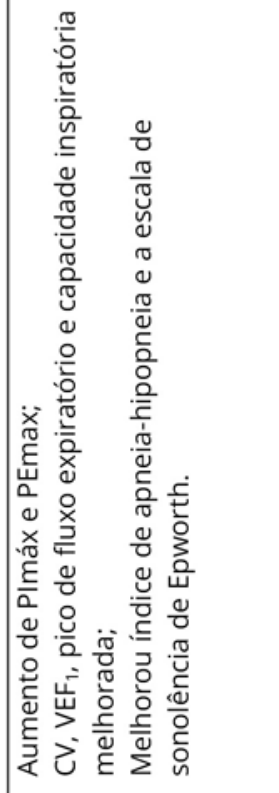 & 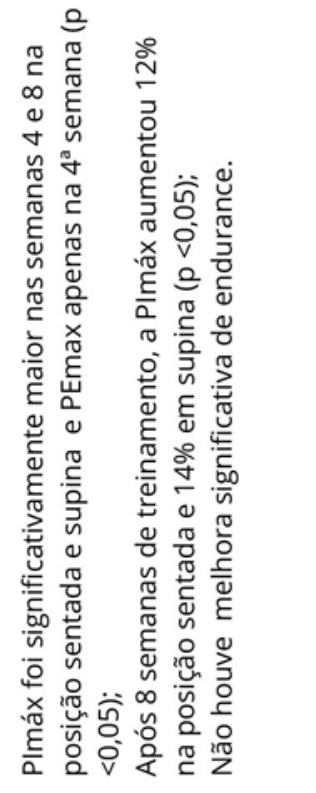 \\
\hline 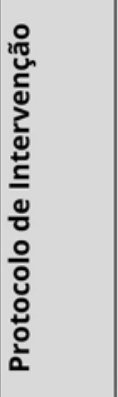 & 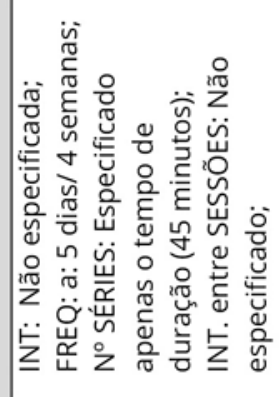 & 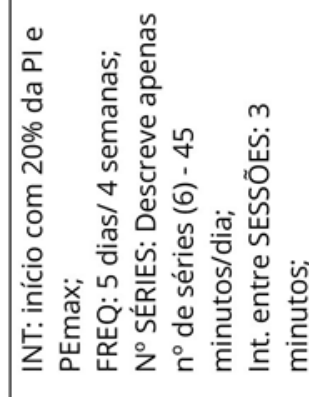 & 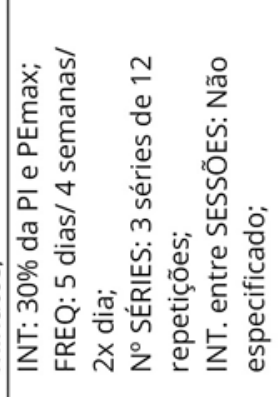 & 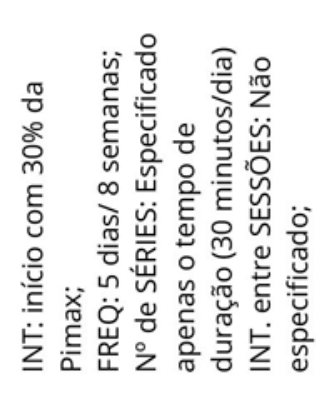 \\
\hline 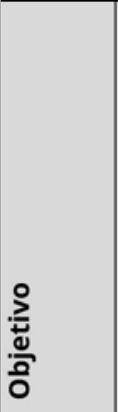 & 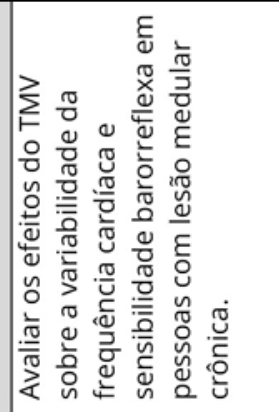 & 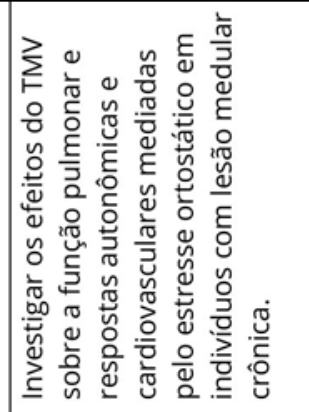 & 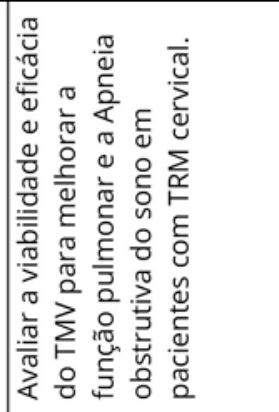 & 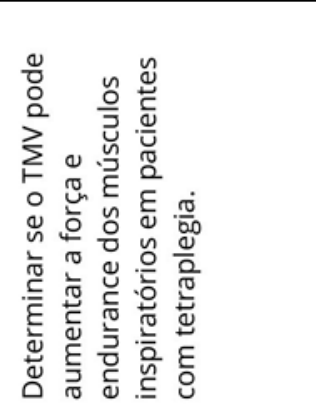 \\
\hline 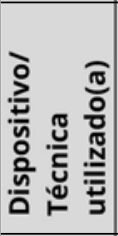 & 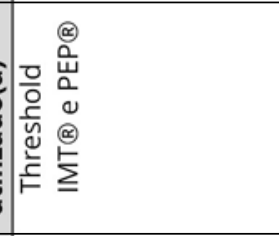 & 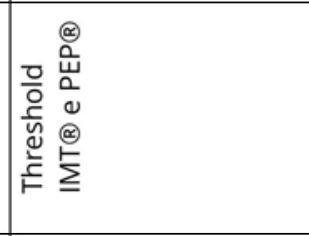 & 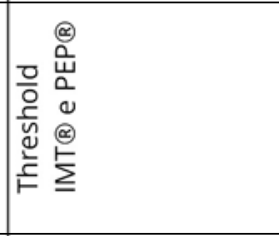 & 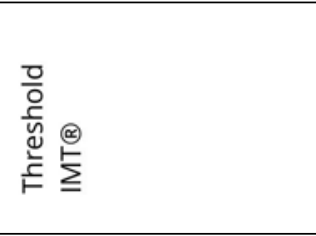 \\
\hline z & 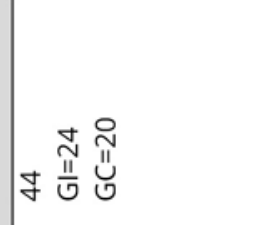 & $\bar{\sim} \overline{\frac{11}{v}}$ & $m$ & $\infty$ \\
\hline 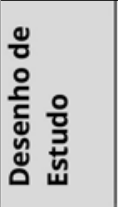 & 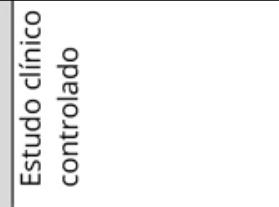 & 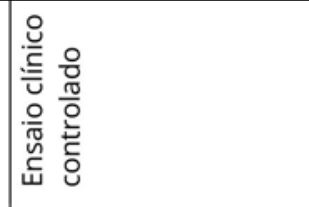 & 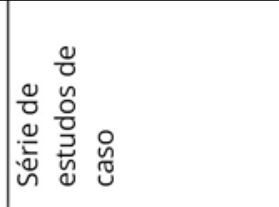 & 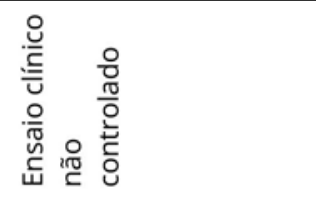 \\
\hline 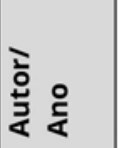 & 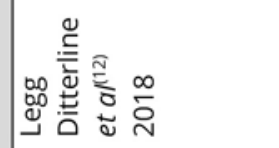 & 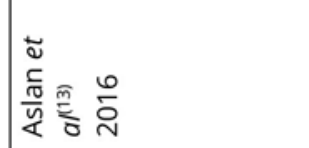 & 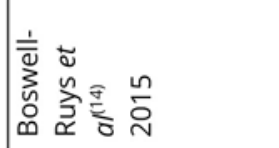 & 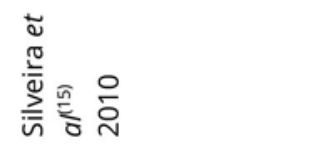 \\
\hline
\end{tabular}




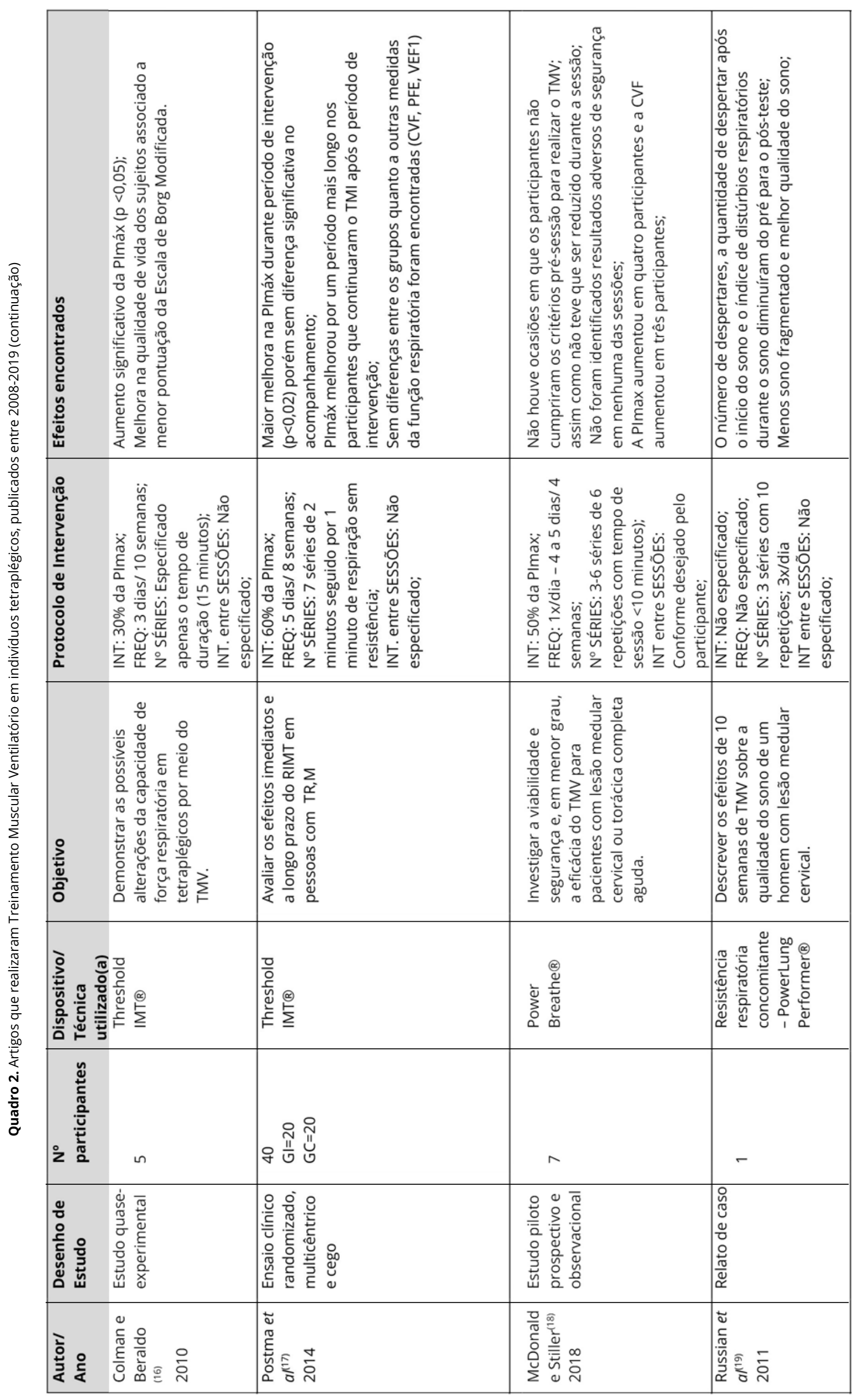

Rev. Pesqui. Fisioter., Salvador, 2020 Maio;10(2):334-345

Doi: 10.17267/2238-2704rpf.v10i2.2809 | ISSN: 2238-2704 


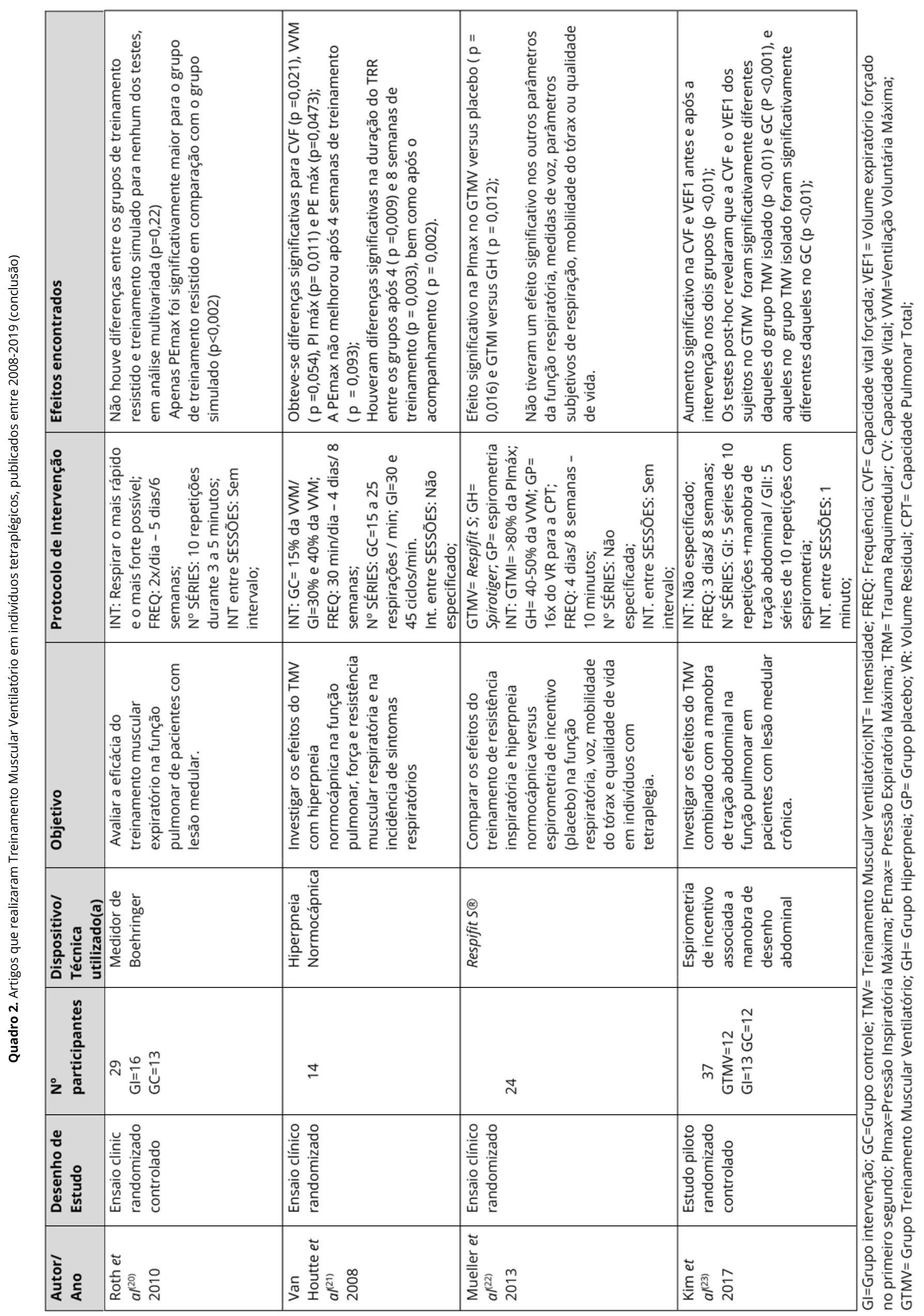




\section{Discussão}

Esta Revisão Integrativa identificou que existem diversos protocolos para o TMV em tetraplégicos pós-lesão medular, utilizando diferentes métodos de forma isolada ou associada. Considerando as evidências de estudos realizados, a literatura relata que de forma geral, a intensidade preconizada para TMV em outros grupos específicos deve ser a maior suportada pelo paciente e que esteja entre 50 a 70\% da PImáx. Em relação à frequência de treinamento, é proposto que seja executado duas vezes ao dia por sete dias na semana, atrelado a um conjunto de cinco séries e seis repetições, totalizando 30 inspirações por sessão, tendo um intervalo de 60 segundos entre cada série ${ }^{8,9,24}$.

Dentre os artigos elegíveis para esta revisão, que descrevem protocolos de TMV com enfoque nos músculos inspiratórios por meio de resistores de carga linear, apenas o estudo de McDonald e Stiller ${ }^{18}$ fez uso da intensidade referente a 50\% da PImax e com progressão dessa carga até $90 \%$ da PImáx. Entretanto, há divergência no que tange a frequência, número de repetições e duração do intervalo, apresentandose menores que o proposto ${ }^{8,24}$. Mueller et al. ${ }^{22}$ utilizaram um protocolo que fazia uso inicial de $80 \%$ da PImáx, mas também apontou diferenças nas outras variáveis, principalmente na redução do tempo de treinamento. Todos os outros autores aplicaram os protocolos com uma intensidade entre 20 e 30\% da PImáx. Somente o trabalho de Boswell-Ruys et al. ${ }^{14}$ foi executado com a frequência proposta de 2 vezes ao dia, mas somente durante 5 dias na semana.

Já no que diz respeito aos parâmetros de número de séries/repetições e intervalo entre as séries, foi observado que a grande maioria não relatou e/ou deixou claro sobre esses aspectos. Ademais, nenhuma descrição de protocolos baseados em evidências foi encontrada na literatura, afim de que pudesse comparar com o que foi proposto pelos autores para treino de músculos expiratórios com carga linear (Threshold $\mathrm{PEP}{ }^{\circledR}$ ) ou não (Medidor de Boehringer), assim como por meio de espirometria de incentivo e manobras associadas. Quanto a Hiperpneia Normocápnica, os protocolos encontrados na literatura sugerem aplicação no mínimo três vezes por semana, com duração média de três meses ${ }^{25}$. Van Houtte et al. ${ }^{21}$ executaram protocolos semelhantes ao preconizado, divergindo apenas na duração menor que três meses. Todavia, essa técnica parece ser limitante quando seu objetivo é o TMV26.
Apesar de outras evidências científicas apontarem a intensidade do TMV entre $50-70 \%$, Boswell-Ruys et al. ${ }^{15}$, Silveira et al. ${ }^{16}$ e Colman et al. ${ }^{17}$, executaram protocolos divergindo do proposto ${ }^{8,9,24}$, utilizando uma intensidade inicial em torno de 30\% da PImax e evidenciaram que o TMV por meio de um resistor de carga linear é eficaz para aumento da força muscular respiratória, obtendo ganhos significativos principalmente em PImax. Colman et al. ${ }^{16}$, apontam que um dos integrantes do estudo alcançou uma das menores respostas, em virtude de número de atendimentos inferior aos demais, sugerindo uma possível influência nas respostas quanto ao período de intervenção, que inclusive pode ser comparado ao estudo de Silveira et al. ${ }^{15}$, onde obtiveram respostas significativas com o tempo de treinamento maior. Outro aspecto importante de ser destacado nesse estudo está relacionado a intervenção precoce em que dois participantes da pesquisa evoluíram de maneira considerável, assim como foi visto nos artigos de McDonald e Stiller ${ }^{18}$ e Mueller et al. ${ }^{22}$ e no qual houve influência positiva nos grupos estudados, sustentando o entendimento de que quanto menor o tempo de lesão, maior é a capacidade do aumento da PImáx ${ }^{27}$.

Mueller et al. ${ }^{22}$ aplicaram um protocolo com intensidade de $80 \%$ da Pimax, apontando efeitos significativos nessa variável e afirmam que há uma forte relação entre a PImáx e capacidade de tosse. Park et al. ${ }^{28}$ mostram que se o volume pré-tosse for insuficiente em virtude da fraqueza muscular inspiratória, a capacidade da tosse diminui. Além disso, uma maior força muscular inspiratória aumenta a tensão diafragmática durante a inspiração e consequentemente proporciona maior recolhimento elástico durante a expiração e, portanto, aumenta o pico de fluxo expiratório (PFE), diretamente proporcional a capacidade de tosse ${ }^{29}$. No entanto, Postma et al. ${ }^{17}$,que utilizaram intensidade de $60 \%$ da Pimax com um tempo maior de treinamento, mostram que o TMI não promoveu alterações significativas a curto, médio e longo prazo, no PFE e no pico de fluxo de tosse (PFT), mesmo apresentando aumento na PImáx, o que pode ser justificado pela variabilidade de níveis de lesão assim como o tempo da injúria medular.

Já Roth e colaboradore ${ }^{20}$ foram os únicos que avaliaram exclusivamente a eficácia do treinamento muscular expiratório, porém não foram encontrados na literatura relatos de proposta de protocolos para esse fim. Contudo, empregando uma intensidade 
subjetiva (respirar o mais rápido e o mais forte), notou-se que apenas os resultados correspondentes a PEmáx foram significativamente maiores no grupo experimental, no entanto, não é surpreendente que tenha sido obtidos ganhos significativos apenas a PEmax, uma vez que o treinamento foi direcionado para a musculatura expiratória. Esse achado traz a reflexão sobre a especificidade do treinamento, umas das bases do TMV ${ }^{8}$. Com esses resultados sugere-se que um protocolo específico para treino de músculos expiratórios pode ter o efeito de aumentar a força de e a eficácia da tosse, promovendo diminuição da retenção de secreções em vias aéreas e, assim, reduzir a ocorrência de pneumonias e outras causas de morbidade respiratória ${ }^{30}$.

Outro princípio do TMV é a reversibilidade da atrofia muscular, este sustenta a idéia que quando o treinamento é interrompido (destreinamento), o corpo se reajusta de acordo com a demanda fisiológica diminuída, e as adaptações benéficas podem ser perdidas ${ }^{8}$. Postma et al. ${ }^{17}$, acompanharam os participantes após o término do treino, com o intuito de registrar permanência ou não dos ganhos. Nesse contexto eles apontam efeito positivo significativo na Pimáx apenas a curto prazo. Talvez para efeitos em longo prazo, seja necessário a manutenção do treinamento, conforme descrito por Weiner et al. ${ }^{31} \mathrm{em}$ pessoas com Doença Pulmonar Obstrutiva Crônica (DPOC).

Ainda abordando sobre os aspectos relativos a função pulmonar, notou-se que o ensaio clínico de Kim et al. ${ }^{23}$ foi o único a utilizar um protocolo de treinamento muscular ventilatório associando espirometria de incentivo a manobra de desenho tração abdominal em pacientes com lesão medular. No entanto, nenhum estudo ainda foi realizado para avaliar os efeitos de um protocolo integrado com ambas as técnicas em tetraplégicos, apesar disso, o estudo aponta melhora da função pulmonar através do aumento da pressão dos músculos abdominais. Mesmo não sendo considerada como uma medida de função pulmonar, os autores acreditam que os músculos abdominais contribuem não apenas para a estabilização da coluna e postura, mas também no que diz respeito a mecânica pulmonar, contudo, a PImáx e a PEmáx não foram avaliadas pré e pós intervenção, sendo que estes parâmetros são importantes para afirmar e quantificar ganhos da performance muscular ${ }^{32}$.
O artigo de Van Houtte et al. ${ }^{21}$ foi o único que relatou o TMV por meio da técnica de hiperpneia normocápnica de forma isolada. A literatura preconiza que o treino por meio dessa técnica não seja a primeira opção de intervenção para TMV, pois se acredita que esse tipo de treinamento aumentaria o risco de fadiga muscular ${ }^{26}$. Contudo, não foram observados nenhum relato de fadiga durante ou após execução do protocolo. Além disso, observou-se que não houve aumento significativo da PEmax, o que difere do encontrado por Roth et al. ${ }^{21}$ através de um protocolo específico para músculos expiratórios.

Quanto aos efeitos do TMV nos parâmetros relacionados ao sono, Russian et al. ${ }^{19}$ apontam mudanças positivas após execução do protocolo. Porém o participante não possuía alguns preditores de distúrbios respiratórios do sono tais como sobrepeso, pescoço grosso e posição de dormir em decúbito dorsal, sendo, portanto, esperado um índice de distúrbio respiratório baixo ${ }^{33,34}$. Notou-se também que o indivíduo realizou exercícios respiratórios não especificados no estudo, no entanto, mesmo associando ao TMV, a PImax não apontou alterações significativas, justificada talvez pelo uso de um dispositivo sem relatos encontrados na literatura de utilização nesse público.

Boswell-Ruys et al. ${ }^{14}$, exploraram variáveis semeIhantes às de Russian et al. ${ }^{19}$ nos itens relacionados aos distúrbios do sono, especificamente a Apneia Obstrutiva do Sono (AOS), mas sabe-se que os problemas inerentes ao sono são muito comuns na população avaliada, embora alguns desses problemas, provavelmente estejam relacionados à alta incidência de apneia central do sono, que é muito mais prevalente nesse público comparada $\mathrm{AOS}^{35}$. Os autores acreditam que efeitos positivos do TMV, nos itens relacionados ao sono podem estar relacionados ao fato dessa intervenção favorecer o treinamento dos músculos dilatadores da via aérea superior, no entanto, esses músculos não são afetados diretamente em lesados medular ${ }^{36}$. Apesar disso, pode-se deduzir que esses protocolos de TMV ${ }^{14,19}$ sejam uma possível alternativa para tratar a AOS e outras desordens do sono, fundamentado na baixa taxa de adesão ao uso do CPAP (pressão positiva contínua nas vias aéreas) nesses indivíduos, já que esta é a ferramenta mais utilizada para esse perfil clínico ${ }^{35}$. 
No que diz respeito a impacto dos protocolos de TMV em questões hemodinâmicas, Aslan et al. ${ }^{13}$ e Legg Ditterline et al. ${ }^{12}$, tiveram objetivos semelhantes e apresentaram os efeitos do TMV sobre a regulação da pressão arterial (PA) assim como sobre a hipotensão ortostática (HO) e sensibilidade barorreflexa. Os efeitos apontados pelos autores está atrelado a hipótese que as oscilações da PA, durante a sessão de TMV podem melhorar e/ou aumentar a elasticidade dos barorreceptores ${ }^{13}$. Contudo, esse hipótese diverge da proposta por Frisbie, onde afirma que as transferências posturais (supino para sentado) gerem oscilações na PA e maior esforço respiratório, aumentando assim, o retorno venoso ao tórax, favorecendo também o aumento do débito cardíaco, uma variável que é de grande valia para a estimulação dos barorreceptores e dessa maneira, responder às flutuações da pressão arterial de maneira mais fisiológica ${ }^{37}$.

A heterogeneidade dos desenhos de estudo bem como dos protocolos aplicados é condizente com os achados de Sheel et al. ${ }^{38}$ e Tamplin e Berlowitz ${ }^{39}$ o que talvez possa ser explicado pelo grande desafio de prestar assistência em saúde a essa população, visto que os mais variados indivíduos podem ser afetados por essa condição, além da divergência dos níveis de lesão, resultando na impossibilidade da generalização dos resultados.

\section{Considerações finais}

Diante desta análise fica evidenciado que protocolos para TMV através de resistores lineares é o mais utilizado em indivíduos com tetraplegia, visto que é também o mais abordado em outros públicos. Além disso, conclui-se que a execução de protocolos com resistores lineares a inspiração, empregando intensidade em torno de 30-60\% da PImax, apresentou efeitos significativos em PImax. No entanto, percebeu-se que há muitas discordâncias quanto as variáveis de treinamento, principalmente intensidade e número de séries propostos. Contudo, é notório que a utilização do TMV em tetraplégicos apresenta certas divergências, principalmente no que diz respeito a escolha das técnicas e/ou dispositivos adequados, já que alguns estudos discorriam sobre TMV utilizando métodos que nunca foram utilizados nessa população.

\section{Contribuições dos autores}

Matos BS, Melo ASM participaram da concepção, delineamento, busca dos dados da pesquisa, interpretação dos resultados e redação do artigo científico. Conceição TMA participou da concepção, delineamento, interpretação dos resultados e redação do artigo científico.

\section{Conflitos de interesses}

Nenhum conflito financeiro, legal ou político envolvendo terceiros (governo, empresas e fundações privadas, etc.) foi declarado para nenhum aspecto do trabalho submetido (incluindo, mas não se limitando a subvenções e financiamentos, participação em conselho consultivo, desenho de estudo, preparação de manuscrito, análise estatística, etc.).

\section{Referências}

1. Eckert MJ, Martin MJ. Trauma: Spinal Cord Injury. Surg Clin North Am. 2017;97(5):1031-1045. doi: 10.1016/j.suc.2017.06.008

2. World Health Organization. Spinal cord injury. [Internet]. 2013. [acesso em 2020 abr. 17]. Disponível em: https://www.who.int/ news-room/fact-sheets/detail/spinal-cord-injury.

3. Kirshblum SC, Burns SP, Biering-Sorensen F, Donovan W, Graves DE, Jha $A$ et al.. International standards for neurological classification of spinal cord injury (Revised 2011). J Spinal Cord Med. 2011;34(6):535-546. doi: 10.1179/204577211X13207446293 695

4. Brunozi AE, Silva AC, Gonçalves LF, Veronezi RJB. Qualidade de Vida na Lesão Medular Traumática. Rev Neurocienc. 2011;19(1):139-144.

5. Kumar R, Lim J, Mekary RA, Rattani A, Dewan MC, Sharif SY et al. Traumatic spinal injury: global epidemiology and worldwide. World Neurosurg. 2018;113:345-363. doi: 10.1016/j. wneu.2018.02.033

6. França ISX, Coura AS, Sousa FS, Almeida PC, Pagliuca LMF. Qualidade De Vida Em Pacientes Com Lesão Medular. Rev Gaúcha Enferm. 2013;34(1):155-163. doi: 10.1590/S198314472013000100020

7. Berlowitz DJ, Wadsworth B, Ross J. Respiratory problems and management in people with spinal cord injury. Breath. 2016;12(4):328-340. doi: 10.1183/20734735.012616

8. Capó-Juan MA. El paciente con lesión medular en fase crónica. Revisión del tratamiento fisioterápico. FisioGlía. 2016;3(1):5-12.

9. Nepomuceno Junior BRV, Gomes-Neto M. Treinamento Muscular Inspiratório no ambiente hospitalar: protocolo para um ensaio clínico randomizado. Revista Pesq. 2016;6(2):158-166. doi: 10.17267/2238-2704rpf.v6i2.896 
10. Nepomuceno Junior BRV, Barreto MS, Almeida NC, Guerreiro $C F$, Xavier-Souza E, Neto MG. Safety and efficacy of inspiratory muscle training for preventing adverse outcomes in patients at risk of prolonged hospitalisation. Trials. 2017;18(1):626. doi: 10.1186/s13063-017-2372-y

11. Botelho LLR, Cunha CCA, Macedo M. O método da revisão integrativa nos estudos organizacionais. Gestão E Sociedade. 2011;5(11):121-136. doi: 10.21171/ges.v5i11.1220

12. Legg Ditterline BE, Aslan SC, Randall DC, Harkema SJ, Castillo C, Ovechkin AV. Effects of Respiratory Training on Heart Rate Variability and Baroreflex Sensitivity in Individuals with Chronic Spinal Cord Injury. Arch Phys Med Rehabil. 2018;99(3):423-432. doi: 10.1016/j.apmr.2017.06.033

13. Aslan SC, Randall DC, Krassioukov AV, Phillips A, Ovechkin AV. Respiratory Training Improves Blood Pressure Regulation in Individuals With Chronic Spinal Cord Injury. Arch Phys Med Rehabil. 2016;97(6):964-73. doi: 10.1016/j.apmr.2015.11.018

14. Boswell-Ruys CL, Lewis CRH, Gandevia SC, Butler JE. Respiratory muscle training may improve respiratory function and obstructive sleep apnoea in people with cervical spinal cord injury. Spinal Cord Ser Cases. 2015;1:15010. doi: 10.1038/ scsandc.2015.10

15. Silveira JM, Gastaldi AC, Boaventura CM, Souza HC. Treinamento de músculos inspiratórios em pacientes com quadriplegia. J Bras Pneumol. 2010; 36(3):313-319. doi: 10.1590/ S1806-37132010000300008

16. Colman ML, Beraldo PC. Estudo das variações de pressão inspiratória máxima em tetraplégicos, tratados por meio de incentivador respiratório, em regime ambulatorial. Fisioter Mov. 2010;23(3):439-449. doi: 10.1590/S0103-51502010000300011

17. Postma K, Haisma JA, Hopman MT, Bergen MP, Stam HJ, Bussmann JB. Resistive Inspiratory Muscle Training in People With Spinal Cord Injury During Inpatient Rehabilitation: A Randomized Controlled Trial. Phys Ther. 2014;94(12):1709-19. doi: $10.2522 /$ ptj.20140079

18. McDonald T, Stiller K. Inspiratory muscle training is feasible and safe for patients with acute spinal cord injury. J Spinal Cord Med. 2019;42(2):220-227. doi: 10.1080/10790268.2018.1432307

19. Russian C, Litchke L, Hudson J. Concurrent respiratory resistance training and changes in respiratory muscle strength and sleep in an individual with spinal cord injury: case report. J Spinal Cord Med. 2011;34(2):251-4. doi: 10.1179/107902611×1297 $\underline{2448729602}$

20. Roth EJ, Stenson KW, Powley S, Oken J, Primack S, Nussbaum SB et al. Expiratory muscle training in spinal cord injury: a randomized controlled trial. Arch Phys Med Rehabil. 2010;91(6):857-61. doi: 10.1016/j.apmr.2010.02.012
21. Van Houtte $S$, Vanlandewijck $Y$, Kiekens $C$, Spengler CM, Gosselink R. Patients with acute spinal cord injury benefit from normocapnic hyperpnoea training. J Rehabil Med. 2008;40(2):11925. doi: $\underline{10.2340 / 16501977-0140}$

22. Mueller G, Hopman MT, Perret C. Comparison of respiratory muscle training methods in individuals with motor and sensory complete tetraplegia: a randomized controlled trial. J Rehabil Med. 2013;45(3):248-53. doi: 10.2340/16501977-1097

23. Kim CY, Lee JS, Kim HD, Lee DJ. Short-term effects of respiratory muscle training combined with the abdominal drawing-in maneuver on the decreased pulmonary function of individuals with chronic spinal cord injury: A pilot randomized controlled trial. J Spinal Cord Med. 2017;40(1):17-25. doi: $\underline{10.1080 / 10790268.2016 .1198576}$

24. Bissett B, Leditschke IA, Green M. Specific inspiratory muscle training is safe in selected patients who are ventilator-dependent: a case series. Intensive Crit Care Nurs. 2012; 28(2):98-104. doi: 10.1016/j.iccn.2012.01.003

25. Silva DSP. Reabilitação respiratória nas pessoas com lesão medular traumática cervical [dissertação]. Coimbra, Portugal: Escola Superior de Enfermagem de Coimbra;2013.

26. Noda JL, Sonoda LT, Sangean M, Fávero FM, Fontes SV, Oliveira ASB. O efeito do treinamento muscular respiratório na miastenia grave: revisão da literatura. Rev Neurocienc. 2009;17(1):37-45. doi: 10.34024/rnc.2009.v17.8602

27. Liaw MY, Lin MC, Cheng PT, Wong MK, Tang FT. Resistive inspiratory muscle training: its effectiveness in patients with acute complete cervical cord injury. Arch Phys Med Rehabil. 2000; 81(6):752-756. doi: 10.1016/s0003-9993(00)90106-0

28. Park JH, Kang SW, Lee SC, Choi WA, Kim DH. How respiratory muscle strength correlates with cough capacity in patients with respiratory muscle weakness. Yonsei Med J. 2010;51(3):392-7. doi: 10.3349/ymj.2010.51.3.392

29. Kang SW, Kang YS, Sohn HS, Park JH, Moon JH. Respiratory muscle strength and cough capacity in patients with Duchenne muscular dystrophy. Yonsei Med J. 2006;47(2):184-90. doi: 10.3349/ymj.2006.47.2.184

30. Estenne M, Troyer A. Cough in tetraplegic subjects: an active process. Ann Intern Med. 1990;112(1):22-28. doi: 10.7326/00034819-112-1-22

31. Weiner $P$, Magadle R, Beckerman $M$, Weiner $M$, BerarYanay N. Maintenance of inspiratory muscle training in COPD patients: one year follow-up. Eur Respir J. 2004;23(1):61-5. doi: 10.1183/09031936.03.00059503

32. Caruso P, Albuquerque ALP, Santana PV, Cardenas LZ, Ferreira JG, Prina E et al. Métodos de diagnóstico para avaliar a força muscular inspiratória e expiratória. J Bras Pneumol. 2015;41(2):110-123. doi: 10.1590/S1806-37132015000004474 
33. Schafer H, Pauleit D, Sudhop T, Gouni-Berthold I, Ewig $\mathrm{S}$, Berthold HK. Body fat distribution, serum leptin, and cardiovascular risk factors in men with obstructive sleep apnea. Chest. 2002; 122(3):829-39. doi: 10.1378/chest.122.3.829

34. Davies RJ, Ali NJ, Stradling JR. Neck circumference and other clinical features in the diagnosis of the obstructive sleep apnea syndrome. Thorax. 1992;47(2):101-5. doi: 10.1136/thx.47.2.101

35. Chiodo AE, Sitrin RG, Bauman KA. Sleep disordered breathing in spinal cord injury: A systematic review. J Spinal Cord Med. 2016;39(4):374-382. doi: 10.1080/10790268.2015.1126449

36. Guimarães KC, Drager LF, Genta PR, Marcondes BF, LorenziFilho G. Effects of oropharyngeal exercises on patients with moderate obstructive sleep apnea syndrome. Am J Respir Crit Care Med. 2009;179(10):962-966. doi: 10.1164/rccm.200806$9810 \mathrm{C}$

37. Frisbie JH. Correction of orthostatic hypotension by respiratory effort. Spinal Cord. 2010;48(5):434-435. doi: 10.1038/sc.2009.148

38. Sheel AW, Reid WD, Townson AF, Ayas NT, Konnyu KJ. Effects of exercise training and inspiratory muscle training in spinal cord injury. J Spinal Cord Med. 2008; 31(5): 500-8. doi: 10.1080/10790268.2008.11753645

39. Tamplin J, Berlowitz DJ. A systematic review and meta-analysis of the effects of respiratory muscle training on pulmonary function in tetraplegia. Spinal Cord. 2014 Mar; 52(3):175-80. doi: $\underline{10.1038 / \text { sc.2013.162C }}$ 